\title{
Functional flexibility in wild bonobo vocal behaviour
}

Zanna Clay, Jahmaira Archbold, Klaus Zuberbuhler

A shared principle in the evolution of language and the development of speech is the emergence of functional flexibility, the capacity of vocal signals to express a range of emotional states independently of context and biological function. Functional flexibility has recently been demonstrated in the vocalisations of pre-linguistic human infants, which has been contrasted to the functionally fixed vocal behaviour of non-human primates. Here, we revisited the presumed chasm in functional flexibility between human and non-human primate vocal behaviour, with a study on our closest living primate relatives, the bonobo (Pan paniscus). We found that wild bonobos use a specific call type ("peeps") across a range of contexts that cover the full valence range (positive-neutral-negative) in much of their daily activities, including feeding, travel, rest, aggression, alarm, nesting and grooming. Peeps were produced in functionally flexible ways in some contexts, but not others. Crucially, calls did not vary acoustically between neutral and positive contexts, suggesting that recipients take pragmatic information into account to make inferences about call meaning. In comparison, peeps during negative contexts were acoustically distinct. Our data suggest that the capacity for functional flexibility has evolutionary roots that predate the evolution of human speech. We interpret this evidence as an example of an evolutionary early transition away from fixed vocal signalling towards functional flexibility. 


\section{Abstract}

13 A shared principle in the evolution of language and the development of speech is the

\section{Functional flexibility in wild bonobo vocal behaviour}

1* Department of Comparative Cognition, University of Neuchatel, Neuchatel, Switzerland; ${ }^{2}$ School of Psychology, University of Birmingham, UK; ${ }^{3}$ School of Psychology \& Neurosciences, University of St Andrews, UK

*Z.Clay@bham.ac.uk; +44 7967567 111; School of Psychology, University of Birmingham, Birmingham, UK, B15 2TT emergence of functional flexibility, the capacity of vocal signals to express a range of emotional states independently of context and biological function. Functional flexibility has recently been demonstrated in the vocalisations of pre-linguistic human infants, which has been contrasted to the functionally fixed vocal behaviour of non-human primates. Here, we revisited the presumed chasm in functional flexibility between human and non-human primate vocal behaviour, with a study on our closest living primate relatives, the bonobo (Pan paniscus). We found that wild bonobos use a specific call type ("peeps") across a range of contexts that cover the full valence range (positive-neutral-negative) in much of their daily activities, including feeding, travel, rest, aggression, alarm, nesting and grooming. Peeps were produced in functionally flexible ways in some contexts, but not others. 
24 Crucially, calls did not vary acoustically between neutral and positive contexts, suggesting

25 that recipients take pragmatic information into account to make inferences about call

26 meaning. In comparison, peeps during negative contexts were acoustically distinct. Our

27 data suggest that the capacity for functional flexibility has evolutionary roots that predate

28 the evolution of human speech. We interpret this evidence as an example of an

29 evolutionary early transition away from fixed vocal signalling towards functional flexibility.

\section{Introduction}

32 A growing body of research suggests that human infant vocal development reveals

33 something about the evolutionary history of language (Tomasello et al., 2005; Locke \&

34 Bogin, 2006; Tomasello, 2008). This is because a basic principle of evolution is that, similar

35 to processes in development, natural selection acts by modifying or adding complexity to

36 existing structures and mechanisms rather than by generating entirely new ones, a logic

37 that has also been applied to the evolution of language (Fitch, 2010). A research goal

38 therefore is to describe the basic design principles of early stages of vocal behaviour, which

39 may have served as building blocks on which subsequent stages of linguistic development

40 have emerged (Oller, 2000; Tomasello et al., 2005; Oller et al., 2013). One of these building

41 blocks is 'functional flexibility', an individual's capacity to produce signals that are

42 detached from a predetermined function to express different psychological states in a

43 range of situations (Griebel \& Oller, 2008; Oller et al., 2013). This is thought to contrast

44 with animal signals and some human vocalisations (e.g. crying, laughter), which are tightly

45 linked to specific psychological and motivational states (Oller et al., 2013). 
47 Part of this reasoning stems from a paucity of evidence of functionally flexible signalling in 48 non-human primates (Oller et al., 2013; Ackermann, Hage \& Ziegler, 2014 but see Lameira 49 et al., 2013). In contrast, recent analyses of prelinguistic human infant vocal interactions 50 showed that three types of 'protophone' vocalisations were used flexibly to express a full 51 range of emotional content (positive, neutral and negative valence) across a range of 52 different situations (Oller et al., 2013). Moreover, some vocal types (squeals, vowel-like 53 sounds and growls) were associated with neutral facial expressions, further suggesting 54 that, in human infants, vocal production can be detached from a specific biological function.

55 This is in contrast to other infant vocalisations, namely crying and laughter, which appear to be fixed to specific affective states. It has therefore been suggested that these types of

57 affect-bound, or functionally fixed, vocalisations resemble non-human primate calls, both in 58 terms of their form and function, as well as the brain mechanisms underlying them

59 (Newman, 1985; Owren, Amoss \& Rendall, 2011; Bryant \& Aktipis, 2014; Owren \& Amoss, 60 2014).

61

62 In animals, functionally fixed signals are often considered equivalent to 'functionally 63 referential' signals, broadly defined as acoustically distinct signals produced in response to 64 a narrow range of stimuli to the extent that a receiver, upon hearing the signal in the 65 absence of the stimuli, responds as if experiencing the eliciting stimuli itself (Macedonia \& 66 Evans, 1993). The alarm calls of numerous primates, for example, have been discussed as 67 being functionally fixed in that they are produced reliably in response to certain classes of 68 predators (such as aerial alarm calls in response to aerial predators) and their underlying

69 affective states (Seyfarth et al. 1980a, b; Zuberbühler, 2003, 2006). While some calls may fit 
70 the notion of functional fixedness, the theoretical assumption that vocalisations must be

71 produced to a narrow range of stimuli in order to functionally refer to something in the

72 world has, in fact, been recently been challenged on the grounds that calls produced to a

73 number of different stimuli may still be functionally referential, given the availability of

74 other contextual cues (Scarantino \& Clay, 2014).

More recent research on animal vocal signalling, particularly by non-human primates, is

77 increasingly suggesting scope for greater flexibility, the spontaneous production of unvoiced, atypical calls by captive great apes being one such example. In many captive facilities, there have been reports of individuals producing atypical, voiceless calls (e.g. "raspberrys") in order to gain the attention of human caregiver, and that some of these are 81 acquired through social learning (e.g. Gorilla gorilla gorilla (Perlman, Patterson \& Cohn, 2012); Pongo pygmaeus (Wich et al., 2009; Hardus et al., 2009; Lameria et al., 2015); Pan troglodytes (Hopkins, Taglialatela \& Leavens, 2007; Taglialatela et al., 2012); Pan paniscus

84 (Taglialatela \& Savage-Rumbaugh, 2003)). Moreover, conditioning experiments with rhesus macaques and cotton-top tamarins have highlighted considerable vocal control, with individuals able to initiate vocalisations and modify various features of their vocal output in response to different external stimuli (Hage, Gavrilov \& Nieder, 2013; Hotchkin,

88 Parks \& Weiss, 2013). Studies of natural communication among conspecifics have also highlighted considerable vocal flexibility. For instance, studies in the contexts of travel recruitment (Gruber \& Zuberbühler, 2013), feeding advertisement (Slocombe et al., 2010;

91 Schel et al., 2013), sexual interactions (Townsend, Deschner \& Zuberbühler, 2008), social 92 greetings (Laporte \& Zuberbühler, 2008) and predator alarms (Crockford et al., 2012; Schel 
93 et al., 2013) have revealed that wild chimpanzees have a notable degree of control over call

94 production and flexibly modify it in response to different audience compositions and, in

95 some cases, in intentional ways (Crockford et al., 2012; Schel et al., 2013). Group-specific

96 'dialects' have also been suggested for four chimpanzee communities living in the same

97 forest in the Ivory Coast (Crockford et al., 2004). In orang-utans, a recent study of the

98 production of variants of an alarm call (the kiss-squeak) in two different wild populations

99 revealed population-specific usage, which indicated a certain degree of arbitrariness

100 (Lameira et al., 2013).

101

102 Research on the communicative behaviour of a human-enculturated and language-trained

103 bonobo ('Kanzi') has already highlighted bonobos as an interesting candidate species to

104 examine flexible primate vocal production (Savage-Rumbaugh \& Lewin, 1994). It has been

105 suggested, for instance, that Kanzi used 'peeps', one of the most common vocalisations in

106 the bonobo repertoire (de Waal, 1988), to communicate with caregivers in ways that

107 conform to conversational rules used in human speech dialogue (Greenfield \& Savage-

108 Rumbaugh, 1990, 1993; Savage-Rumbaugh, 1990, 1998; Segerdahl, Fields \& Savage-

109 Rumbaugh, 2005). Another study also suggested that Kanzi's vocal repertoire had become

110 augmented to include novel acoustic variants of the 'peep' vocalisation produced in

111 response to different food types and that, astonishingly, the acoustic structure of these

112 vocal variants resembled the corresponding spoken English words used by his caregivers

113 (Hopkins \& Savage-Rumbaugh, 1991; Taglialatela \& Savage-Rumbaugh, 2003). 
115 While Kanzi's communicative behaviour is suggestive of considerable communicative

116 flexibility, research into flexibility in the natural vocal communication system of bonobos

117 remains scant (Liebel et al., 2013). The bonobo vocal repertoire has been described as

118 highly graded, containing up to fifteen principal call types (de Waal, 1988; Bermejo \&

119 Omedes, 1999). Consistent with the more traditional view of primate vocal signalling, most

120 bonobo call types appear to be tied to particular emotional states or valence classes, such

121 as pant-laughing during socio-positive interactions, screaming and pout-moaning in

122 response to agonism, threat barks during aggressive attacks, pant-grunts during

123 submissive greetings and alarm barks in response to predators (de Waal, 1988; Clay et al.,

124 Unpublished Data). However, as indicated in the Kanzi studies, their most frequent vocal

125 type, the 'peep', is produced across an array of behavioural contexts (Fig 1., (de Waal,

126 1988)). Analyses of the bonobo vocal repertoire in the wild (Bermejo \& Omedes, 1999) and

127 in captivity (de Waal, 1988) both stressed the importance of the peep in bonobo

128 communication and reported its highly flexible and varied use across different behavioural

129 contexts.

130

131 Although peeps are produced across an array of contexts, they are especially common

132 during feeding events and, consequently, have so far only been systematically studied as a

133 food-associated signal (van Krunkelsven et al., 1996; Clay \& Zuberbühler, 2009, 2011). For

134 instance, peeps produced in response to food discovery have been shown to be frequently

135 combined with other call types into longer sequences, whereby the probabilistic

136 organisation of the sequence conveys information to receivers about perceived food quality

137 (Clay \& Zuberbühler, 2009, 2011). 
139 The apparently varied usage of the peep vocalisation in both natural and artificial settings

140 suggests it to be an interesting candidate to study evidence for vocal flexibility. Moreover,

141 promising findings from studies of Kanzi indicated that peeps may even have the potential

142 to be used in language-like ways, such as conforming to conversational rules (Greenfield \&

143 Savage-Rumbaugh, 1990, 1993; Savage-Rumbaugh, 1990, 1998; Segerdahl, Fields \&

144 Savage-Rumbaugh, 2005) and being modified into context-specific acoustic variants that

145 correspond to spoken human words (Taglialatela \& Savage-Rumbaugh, 2003). Despite

146 converging reports indicating flexibility in this vocalisation, to our knowledge, no

147 systematic investigation of their general use has been conducted. For example, it is

148 currently unknown whether peeps share the same acoustic structure across contexts or

149 whether they are context-specific. Before investigating the degree to which this candidate

150 call type may be produced flexibly, it is important to first establish whether their acoustic

151 structure varies across contexts.

152

153 The aim of the current study is therefore to systematically analyse the acoustic structure of

154 peeps to assess whether they are tied to specific behavioural contexts or whether, like

155 human infants (Oller et al., 2013), bonobos are capable of producing the same vocalisation

156 across a range of valence states. From an evolutionary perspective, looking for non-human

157 primate vocalisations that are not so tightly tied to biological function but are used in more

158 functionally flexible ways provides potentially relevant insights for the evolution of human

159 speech. If humans are the only species capable of functional flexibility, that is, to produce

160 the same vocalisation across different valence states, peeps should be expected to vary 
161 according to the valence (i.e. from positive to neutral to negative) of the contexts. To this

162 end, we analysed various acoustic parameters of bonobo peeps produced in different

163 behavioural contexts relating to the three principal valence dimensions (positive-neutral-

164 negative) to explore whether these structural parameters provided acoustic cues relating

165 to the inferred affective valence. This approach differs from the 'discrete emotion'

166 approach, which presupposes discrete emotional states in animals and humans that arise

167 in response to anticipation of rewarding or punishing events, such as fear or pleasure

168 (Russell, 1980; Mendl, Burman \& Paul, 2010). By taking a more graded approach, the

169 current study is better suited for making direct comparisons with previous research on

170 prelinguistic human infant vocalisations (Oller et al., 2013).

171

172 Materials \& Methods

173

174 Behavioural observations were conducted on individuals from the Bompusa community of

175 wild bonobos by Z.C. from October 2013 to March 2014 at Lui Kotale, located near the

176 Salonga National Park, in DR Congo. At this time, the fully habituated and fully identified

177 community consisted of twelve adult females, two subadult females, five adult males two

178 subadult males and eighteen immatures (juveniles and infants). Animal focal animal

179 sampling (15 minutes) was conducted on all males and all adult females throughout the

180 study period, amounting to an average of 11 focal hours per individual.

181

182 We recorded vocalisations produced by focal individuals across a variety of behavioural

183 contexts, as they occurred. We recorded vocalizations at distances of 7-20 m using a 
184 Sennheiser MKH816T directional microphone and Marantz PMD661 solid-state recorder

185 (Microphone frequency response: $50-20000 \mathrm{~Hz}, \pm 3.5 \mathrm{~dB}$; sampling rate of $44.1 \mathrm{kHz}, 16 \mathrm{bits}$ 186 accuracy).

187

188 Our acoustic analyses focussed on the bonobo peep, a high-frequency, closed mouth 189 vocalisation (approx. 2,200 Hz, de Waal, 1988, see Fig. 1), short in duration (approx. $0.1 \mathrm{sec}$ 190 (de Waal, 1988, Clay \& Zuberbühler, 2009)) and characterised by a simple, flat acoustic

191 form composed of several harmonics that are generally un-modulated. In order to analyse

192 the peep structure across different contexts, we first identified the most vocally active 193 individuals from focal recordings, identifying those that produced vocalisations in at least

194 two feeding contexts and two non-feeding contexts, which resulted in a sample of eight 195 individuals (four adult males, one subadult male and three adult females). Behavioural 196 contexts were mutually exclusive, i.e. peeps produced holding or consuming food while 197 travelling or resting were excluded. In order to compare the acoustic structure of peeps in 198 different contexts we compared the acoustic structure of peeps during the contexts that 199 generated the most peep vocalisations per individual. We collected peeps produced at the 200 onset of each behavioural context (i.e. at the onset of food discovery or travel). Because the 201 number of peeps produced at the vocal sequence onset varied across different calling 202 events, we analysed up the first three consecutive peeps produced at the beginning of a 203 vocal event by the same individual as this was the typically number of peeps produced in a 204 consecutive sequence. We calculated mean scores per parameter across the three peeps to 205 standardise across the same calling event. 
207 In contrast to an analysis focussing on discrete emotional states, we were interested in first 208 establishing whether bonobo vocalisations may be used flexibly across the valence contexts 209 (positive-neutral-negative), as has been demonstrated in prelinguistic infants (Oller et al., 210 2013). Therefore, for each individual, we randomly selected a balanced sample of eight

211 peep recordings produced during feeding contexts (feeding on shoots/seeds on the ground 212 and fruits in trees), which we inferred to be as approximately positive in overall valence

213 (Briefer, Tettamanti \& McElligott, 2015) and eight peep events produced during non-

214 feeding contexts (resting and travel), which we inferred to be, in comparison to feeding,

215 relatively neutral in valence. In order to capture the spectrum of emotional valence in our

216 acoustic analyses (i.e. positive-negative-neutral), we also analysed a sample of peeps

217 associated with predator alarm responses and in response to agonistic interactions as the

218 victim, which were both taken to represent negative valence. As peeps in response to

219 agonistic and alarm contexts were rare, we analysed a balanced and randomized sample of 2204 peep samples per individual, taken from independent behavioural events produced by 7

221 of the original 8 individuals $(\mathrm{N}=28$ in total). The eighth individual was excluded in this

222 sample due to inadequate sample size. This amounted to a total of 128 peep events. We

223 selected two contexts per valence class (positive-negative-neutral) in order to maximise

224 sample size as well as to adequately capture the potential acoustic variation in different

225 contexts. In order to capture variation in the feeding experience overall, recordings from

226 feeding contexts included a randomized and balanced selection of vocal events in response

227 to feeding on fruits in trees as well as to herbaceous shoots on the ground. For non-feeding

228 contexts, we analysed a randomized sample of recordings for each individual produced

229 during rest and travel on the ground. For negative valence contexts, we analysed a 
230 randomised balanced sample of peeps produced during agonistic conflicts and predator

231 alarm contexts.

232

233 We carried out all quantitative acoustic analyses with Praat 5.4.01 using the following

234 settings: analysis window length $0.05 \mathrm{~s}$, dynamic range $70 \mathrm{~dB}$; pitch range $500-3000 \mathrm{~Hz}$,

235 optimized for voice analysis, spectrogram view range $0-10 \mathrm{kHz}$. We performed pitch

236 analysis using a script ("Analyse Source Editor") written by M. Owren (personal

237 communication). We then took the following spectral measurements from the fundamental

238 frequency (F0): 1) mean fundamental frequency (Hz): average F0 across the entire call; (2)

239 frequency at call onset, (3) frequency at call middle; (4) frequency at call offset; (5)

240 transition onset $(\mathrm{Hz})$ : frequency of maximum energy at call onset minus frequency of

241 maximum energy at call middle; (6) transition offset (Hz): frequency of maximum energy at

242 call middle minus frequency of maximum energy at call offset; (7) maximum fundamental

243 frequency $(\mathrm{Hz})$ : maximum frequency of F0; (8) minimum fundamental frequency (Hz):

244 minimum frequency of F0; (9) number of harmonics: number of harmonic bands visible. In

245 the temporal domain, we measured the call duration (10).

247 Next, we screened the data for outliers by producing standardized Z scores, rejecting any

248 calls with a Z score greater than 3.29 in one or more parameters (Tabachnick \& Fidell,

249 2001). We regressed all parameters to check for multi-colinearity and singularity,

250 removing parameters with a variance inflation factor greater than 10 . We then conducted a

251 Discriminant Function Analysis (DFA) to assess whether the uncorrelated acoustic

252 variables could discriminate between different behavioural contexts. Each of the eight 
253 individuals equally contributed eight randomly selected calls for both food (henceforth 254 'positive valence') and non-food (henceforth 'neutral valence') contexts and four calls per

255 individual were entered for the negative valence ( $\mathrm{N}=156$ peep samples in total). To cross-

256 validate the discriminant functions produced in the analysis, we used the leave-one-out

257 classification procedure, which classifies each calls by the functions derived from all calls

258 other than that one. We used Binomial tests to analyse whether the proportion of correct

259 discrimination differed significantly from chance.

261 In order to examine whether peeps conveyed information about caller identity, we

262 conducted a DFA using the same data used for the above analysis but taking individual

263 identity as the discriminating factor. We additionally conducted separate DFAs for the

264 positive and neutral valence contexts in order to control for behavioural context. We were

265 unable to include separate DFAs for the negative valence context due to small sample size

$266(\mathrm{~N}=4$ calls per individual) compared to the number of acoustic parameters under scrutiny,

267 which led to inadequate statistical power.

268

269 Since the acoustic data were two-factorial (caller ID; context), it has been argued that

270 conventional DFA does not allow for a valid estimation of the overall significance of

271 discriminability (Mundry \& Sommer, 2007). Therefore, for any significant DFA

272 discrimination, we conducted a permuted Discriminant Function Analysis (pDFA), using a

273 macro written by R. Mundry and C. Sommer (Mundry \& Sommer, 2007; R. Mundry,

274 Personal Communication). The pDFA estimates the significance of the number of correctly

275 classified calls (cross-validated), taking into account repeated contributions per individual 
276 caller.

277

278 Following significant discrimination in the pDFA and diagnostic tests, we used Univariate 279 Analyses of Variance tests to explore whether each of the acoustic parameters varied 280 statistically with context, entering Caller Identity as a Random Factor and Context as the 281 Fixed Factor.

282

283 All statistical tests were carried out using SPSS version 21.0 (SPSS Inc., Cary, NC, U.S.A.) and 284 R Studio version 3.1.1 (The R Foundation for Statistical Computing, Vienna, Austria). All 285 tests were two tailed and alpha levels were set at 0.05 , unless stated as being corrected. We 286 applied standardised Bonferroni corrections for multiple comparisons.

288 Results

289 During focal animal sampling, we recorded peeps in response to over a dozen different 290 behavioural contexts, which, across all focal individuals, included feeding on fruits, leaves, 291 seeds, flowers in trees and on shoots, seeds, leaves and fruits on the ground. It also 292 included travelling, resting, grooming, preparing a nest, interacting sexually, responding to 293 vocalisations from other parties, descending from trees after feeding, alarm responses to

294 predators or unexpected events, weather changes, agonistic interactions, submissive or 295 appeasement responses towards more dominant individuals, and vocal greetings to the 296 arrival of another individual into the party. 297

298 Acoustic structure of peeps 
299 We compared the acoustic structure of peeps produced in different contexts (feeding;

300 travel/rest; agonism/alarm) that were associated with different emotion valences

301 (positive; neutral; negative valence, respectively), Fig. 1 and that generated the most peep

302 vocalisations across individuals. Following a multi-colinearity screening, we entered six of

303 the nine original acoustic parameters into our acoustic analyses for eight individuals (total

$304 \mathrm{~N}$ call events = 156: call duration, mean F0, F0 at call onset, number of harmonics,

305 transition onset and transition offset) and applied logarithmic transformations on three of

306 the acoustic parameters to improve their homogeneity of variance. Results from a cross-

307 validated discriminant function analysis revealed that while the DFA model generated two

308 significant discriminant functions (Wilks Lambda: 0.550, $\left.\chi^{2}(\mathrm{df}=14)=80.007, \mathrm{P}<.001\right)$,

309 peeps produced in association with positive valence contexts could not be reliably

310 discriminated from those produced in all other contexts: the functions only classified

$31149.3 \%$ of the calls correctly, which was below chance level (Binomial test $(0.14) \mathrm{P}>0.05)$.

312

313 On a pairwise basis, DFA analyses further revealed that peeps produced in association with

314 positive valence contexts could not be reliably discriminated from those produced during

315 neutral valence contexts (Wilks Lambda: $0.947, \chi^{2}(\mathrm{df}=6)=6.638, \mathrm{P}=0.356$ ). In a cross-

316 validated analysis, the functions only classified $52.3 \%$ of the calls correctly, which was

317 below chance level (Binomial test (0.5) P > 0.05). However, peeps associated with negative

318 valence (i.e. alarm and agonism) could be significantly discriminated from those produced

319 in association with feeding (82.1\% of calls correctly classified; Wilks lambda $=0.468, \chi^{2}$ (df

$320=7)=59.602, \mathrm{P}<.001 ;$ Binomial test $(0.5) \mathrm{P}<.001$, Bonferroni corrections), which was

321 validated in a subsequent pDFA controlling for repeated contributions $(\mathrm{P}=0.009)$. 
322 Similarly, there was significant discrimination of peeps produced in response to negative

323 valence contexts to those during neutral valence contexts, with $77.4 \%$ of calls (cross-

324 validated) correctly classified (Wilks Lambda $=0.551, \chi^{2}(\mathrm{df}=6)=47.107, \mathrm{P}<.001$;

325 Binomial test $(0.5) \mathrm{P}<.001$, Bonferroni corrections).

326

327 Caller Identity

328 We used the same cross-validated DFA procedure to test whether peeps could be

329 acoustically discriminated on the basis of caller identity ( $\mathrm{N}=8$ individuals). The model

330 generated six significant discriminant functions (Wilks Lambda: $0.371, \chi^{2}(\mathrm{df}=42)=$

$331119.043, \mathrm{P}<.001$ ), which discriminated caller identity at a significantly higher rate than

332 chance (cross-validated correct classification: 31.3\%, Binomial test $(0.125) \mathrm{P}<0.001)$.

333

334 We then conducted two separate DFAs to examine individual identity discrimination for

335 peeps in positive and neutral contexts. Results from the analyses were equivalent, with

336 identity significantly discriminated in both contexts (Individual identity in Feeding

337 contexts $31.3 \%(20 / 64)$ calls correctly classified: Wilks Lambda $=0.234, \chi^{2}(\mathrm{df}=42)=$

338 81.285, $\mathrm{P}<0.001$; Binomial test $(0.125) \mathrm{p}<0.001$; in non-feeding contexts: $32.8 \%(21 / 64)$

339 calls correctly classified Wilks lambda $=0.210, \chi^{2}(\mathrm{df}=42)=87.313, \mathrm{P}<0.001$; Binomial

340 test $(0.125) \mathrm{P}<.001)$.

341

342 Comparing acoustic parameters

343 At the level of acoustic parameters, Univariate ANOVAs (Caller Identity as a random factor)

344 revealed that the mean call duration, the mean fundamental frequency and the mean 
345 frequency at call onset varied significantly as a function of behavioural context (Mean call 346 duration $F_{2,12}=5.625, P=0.019 ;$ Mean F0: $F_{2,12}=19.054, P<.001 ; F 0$ call onset: $F_{2,12}=$

347 40.259, $\mathrm{P}<0.001$ ). Pair-wise comparisons (standard Bonferroni corrections), as shown in

348 Figure 2, of fundamental frequency (F0) parameters showed that peeps produced in

349 association with negative valence had a significantly higher mean F0 and a higher onset F0

350 compared to peeps associated with positive valence $\left(\right.$ Mean $\mathrm{F}_{\text {negative }}=2131 \pm 267 \mathrm{~Hz}$, Mean

$351 \quad \mathrm{~F}_{\text {positive }}=1660 \pm 133 ; \mathrm{F}_{1,6}=16.862, \mathrm{P}=0.006 ; \mathrm{F} 0$ at call onset negative $=2027 \pm 194 \mathrm{~Hz}, \mathrm{~F} 0$ at

352 call onset $_{\text {positive }}=1612 \pm 125 \mathrm{~Hz} ; \mathrm{F}_{1,6},=35.990, \mathrm{P}=0.001$ ) and neutral valence (Mean

$353 \quad \mathrm{~F}_{\text {neutral }}=1584 \pm 210 \mathrm{~Hz}: \mathrm{F}_{1,6}=27.160, \mathrm{p}=0.002 ; \mathrm{F} 0$ at call onset $_{\text {neutral }} 1508 \pm 186 \mathrm{~Hz}, \mathrm{~F}_{1,6}=$

$35469.887, \mathrm{P}<.001)$. Although peeps associated with negative valence were shorter in

355 duration compared to those associated with positive valence $\left(\right.$ Mean call duration $_{\text {negative }}=$

$3560.12 \pm 0.14$, mean call duration ${ }_{\text {positive }}=0.15 \pm 0.03: \mathrm{F}_{1,6}=8.316, \mathrm{P}=0.028$ ), the result was

357 not significant under the Bonferroni correction. There were no other significant acoustic 358 differences.

\section{Discussion}

361 Results suggest that, contrary to current models, humans may not be unique among

362 primates in their ability to produce functionally flexible vocalisations. Our acoustic

363 analyses suggest that the 'peep' calls of wild bonobos are produced in such flexible ways in

364 response to a range of different behavioural contexts of varying affective valence.

365 Following similar evidence in pre-linguistic human infants (Oller et al., 2013), bonobos

366 produced peep vocalisations across a range of neutral, negative and positive contexts in

367 different behavioural situations. Although peeps produced in negative contexts differed 
368 acoustically, the acoustic structure of peeps produced in association with positive valence

369 (feeding), could not be discriminated from neutral valence (travel, rest), despite the fact

370 that behavioural contexts were mutually exclusive. Specifically, peeps produced in

371 association with negative valence (alarm and agonism) possessed significantly higher mean

372 fundamental frequencies, higher frequencies at call onset and shorter durations. The

373 finding of acoustic variants within the same call type across different contexts is a relatively

374 common finding in studies of primate vocal behaviour (Owren et al., 1997; Rendall et al.,

375 1999; Rendall, 2003; Slocombe \& Zuberbuhler, 2007). Moreover, the fact that context-

376 specific acoustic cues were found in negative but not other contexts has interesting

377 implications both at the level of call production and evolutionary function. In terms of call

378 production, the acoustic differences (higher frequencies and shorter call duration) found

379 for negative contexts are most likely a direct result of higher subglottal air pressure during

380 call production (Fitch \& Hauser, 1995; Fitch, 2006), probably because individuals perceive

381 situations as charged, tense and urgent. This is likely to have direct physiological

382 consequences such that the same volume of air is passing through the vocal tract but at a

383 higher speed so that the vocal folds oscillate at a higher frequency for a shorter period of

384 time.

385

386 The fact that peeps produced during negative contexts are more constrained by the

387 physical mechanics of vocal production raises the possibility that, in the course of language

388 evolution, functional flexibility may first have occurred in positive and neutral contexts.

389 Generally, cues to emotional valence are mechanistically harder to conceal during negative

390 contexts, which impedes the vocal control that is required for functional flexibility. 
391 Currently, it is not known whether the functionally flexible calls of human infants also vary

392 acoustically as a function of affect valence, as no acoustic analyses have been conducted

393 (Oller et al., 2013). Our prediction is that, even in humans, negative emotional valence is

394 equally conveyed in the acoustic structure of human vocalisations, including infant calls.

395

396 Overall, our results suggest that bonobo peep production may represent a somewhat

397 intermediate stage between functionally fixed (as seen for most primate vocalisations) and

398 functionally flexible signals, as seen in most human vocalisations (Oller et al., 2013).

399 Nevertheless, peeps could be reliably discriminated on the basis of caller identity across

400 contexts, suggesting that the calls reliably convey other relevant information to receivers.

401

402 In order to make comparisons with evidence from pre-linguistic human infants (Oller et al.,

403 2013), our study took a 'dimensional approach' to emotions (Russell, 1980; Watson et al.,

404 1999; Mendl, Burman \& Paul, 2010) by focussing on the valence associated with the

405 eliciting behavioural contexts. According to the 'dimensional approach', emotions are

406 characterized along two core dimensions: valence (negative or positive; e.g. sad versus

407 happy; Russell, 1980) and arousal (e.g. calm versus excited (Mendl, Burman \& Paul, 2010).

408 This contrasts with the 'discrete emotions' approach (Ekman, 1992; Panksepp, 1998),

409 which although valuable for examining specific emotions, has been suggested to over-focus

410 on certain emotions and not others (e.g. positive ones (Boissy et al., 2007), as well as

411 lacking an overall framework that integrates a wider range of emotional states (Mendl,

412 Burman \& Paul, 2010). The 'dimensional approach' has been shown to be more powerful

413 for studying animal emotions (Mendl et al., 2009; Mendl, Burman \& Paul, 2010) across 
414 behavioural (Briefer, Tettamanti \& McElligott, 2015; Reefman, Weschler \& Gygax, 2009;

415 Imfeld-Mueller et al., 2011), physiological (da Costa et al., 2004; Davies, Radford \& Nicol,

416 2014), and cognitive domains (Nettle \& Bateson, 2012; Briefer \& McElligott, 2013).

417 Consequently, the current study is therefore unable to provide more detailed insights into

418 the relationship between vocal production and discrete emotional states, such as fear or

419 pleasure (Ekman, 1992; Panksepp, 1998). It can be assumed that considerable variation in

420 discrete emotional states most likely exists within the contexts recorded here, for instance,

421 feeding may elicit different discrete emotional states according to other related factors (e.g.

422 social rank, age, group size). A substantial follow-up study, involving a greater sample size

423 of both calls and contexts, that also controls for potentially confounding factors, would be

424 needed to address the vocal indicators of discrete emotional states.

425

426 It has been suggested that the presence of functionally flexible vocalisations in pre-

427 linguistic human infants is evidence for an evolutionary divergence towards speech

428 production and variation in the expression of emotion across different utterances that sets

429 humans apart from the rest of the primate lineage (Oller et al., 2013). For instance, these

430 vocalisations, known as 'protophones' are produced across a full range of affect states and

431 were the most commonly occurring vocal type, suggesting that even pre-linguistic infants

432 possess considerable 'exploratory vocal freedom' and utilise a communication system that

433 is predominantly detached from function. Protophones were most commonly associated

434 with neutral facial expressions compared to cry and laughter, which were tightly linked to

435 negative and positive affect, respectively, further highlighting their greater emotional

436 detachment. Interestingly, bonobo peeps are also typically produced with a neutral facial 
437 expression, produced while the mouth remains closed without any particular facial 438 expression (de Waal, 1987).

440 As always, it is somewhat problematic to draw firm conclusions from negative evidence,

441 that is, the fact that we found no acoustic differences across non-negative valence contexts.

442 For example, it is possible that there was subtle variation in other acoustic parameters that

443 we did not analyse, such as amplitude modulation (Fichtel \& Hammerschmidt, 2001). Our

444 recordings were made under natural conditions with free-ranging animals, which led to

445 various constraints, such as recording at varying distances, directions and atmospheric

446 conditions, which made it impossible to derive reliable measures of amplitude modulation.

447 Future research on peeps using a larger sample size in more controlled acoustic conditions

448 could address this issue. More conclusive evidence would require carefully designed

449 playback experiments to test whether bonobos have more difficulties discriminating

450 positive and neutral peeps, compared to negative peeps. This is important because the

451 notion of functional flexibility also makes predictions about signaller-receiver interactions,

452 i.e. between a signaller's communicative act, or 'illocutionary force', and a recipient's

453 consequential response, or 'perlocutionary effect'. For example, an infant's "complaints"

454 may be associated with specific vocalisations showing negative affect, which then induces a

455 caregiver offering to feed after interpreting the negative affect vocalisation as an indicator

456 of hunger (Austin, 1975; Bates et al., 1979, Oller et al. 2013). The interaction between

457 signaller and receiver would be a relevant aspect to explore in future work, such as using

458 match to sample experiments or naturalistic playback studies. 
460 While functional flexibility is undoubtedly more developed in human speech, results from

461 the current study contribute to the debate surrounding speech evolution by highlighting

462 evidence for a possible intermediate stage in the communicative repertoire of our closest

463 living relative, the bonobo. While much of bonobo vocal behaviour is functionally fixed by

464 nature, such as screaming during agonistic encounters, barking during alarm and laughing

465 during play (de Waal, 1988), their most common vocalisation, the 'peep' is also produced in

466 highly flexible ways across a wide range of contexts and valence states. Previously, the

467 peep vocalisation had been primarily considered as a food-associated call that was

468 combined with other calls in a sequence in order to convey information about food quality

469 (de Waal, 1988; Clay \& Zuberbühler, 2009, 2011). It is possible that when combined in a

470 particular way with other calls, or when combined with a certain context, peeps may still

471 functionally refer to specific events in the external world (Scarantino \& Clay, 2014). Future

472 research, probing the manner in which peeps are combined with other calls and contextual

473 stimuli, is needed to address this question.

474

475 It is relevant to note that studies of language-competent bonobos have already highlighted

476 bonobos as a good model for studying the prerequisites for language evolution (Hopkins \&

477 Savage-Rumbaugh, 1991; Taglialatela \& Savage-Rumbaugh, 2003). In addition to their

478 remarkable ability to acquire human and artificial language systems in captivity (e.g.

479 Segerdahl, Fields, \& Savage-Rumbaugh, 2005), bonobo vocal behaviour has been suggested

480 to be more flexible and dialogue-like than chimpanzees. An interesting possibility is that

481 peeps function to draw attention to and "comment" on novel items or environmental

482 events (de Waal, 1988; Savage-Rumbaugh, Shanker \& Taylor, 1998), a communicative 
483 behaviour also found in early language development (Tomasello \& Carpenter, 2007).

484 Future research should determine whether bonobos are also capable of varying the

485 expression of emotional valence across different utterances within the same vocal event

486 using the same vocal type.

487

488 An alternative possibility is that the production of peeps fits within a more generalised

489 function that extends across multiple contexts (Notman \& Rendall, 2005). For instance, it

490 may have a broader social function related to cohesion or spacing which may explain

491 patterns of usage across seemingly disparate behavioural contexts. For example, the

492 vocalisations produced during feeding by dolphins (Janik, 2003) and greater-spear nosed

493 bats (Wilkinson \& Boughman, 1998) have been suggested to function to coordinate social

494 foraging rather than being specific to feeding per se. The rationale for cohesion effects may

495 even be supported by the current results regarding negative contexts, since cohesion in this

496 case could be outweighed by the urge to escape a predator or a conspecific.

Another proximate explanation for shared acoustic structure is an underlying a shared motivational aspect to the eliciting contexts in question. To address this issue, a solid

500 framework to assess expressions of affect in great apes is first required. One approach, 501 already employed for human infants (Oller et al., 2013), could involve comparing affect 502 expression in corresponding modalities, such as coding facial expressions produced in 503 association with the vocalisation (e.g. Parr et al., 2008; Parr, Waller \& Vicks, 2007).

504 However, unlike the facial affect analyses conducted in association with vocalisation 505 production for human infants (Oller et al., 2013), bonobo peep vocalisations are closed- 
506 mouth vocalisations, making facial affect coding less appropriate. Future work using

507 associated playbacks and hormonal analyses may also provide relevant insights into

508 underlying motivational states during vocal production (Mateo, 2010).

509

510 In summary, the current study contributes promising insights into the evolution of human

511 speech by suggesting an intermediate stage between fully-fledged functional flexibility in

512 human speech production and the more traditionally viewed of fixed signals of non-human

513 primates. Rather than being a uniquely human trait, results from the current study reflect a

514 growing body of literature that suggests that flexible vocal signalling (e.g. Lemasson et al.,

515 2005, 2011; Ouattara, Lemasson \& Zuberbühler, 2009; Crockford et al., 2012; Koda et al.,

516 2013; Liebel et al., 2013) and perhaps even functional arbitrariness (Lameria et al., 2013)

517 may have deeper roots in the primate lineage than previously assumed. By demonstrating

518 the potential for functional flexibility in the vocal behaviour of a great ape species, the

519 results provide a useful springboard from which evidence for functional flexibility in other

520 animal species and across different modalities can be explored.

521

522 Acknowledgements

523 We thank Gottfried Hohmann for granting us permission to conduct research at Lui Kotale

524 and for ongoing support and advice. We are grateful to the Institut Congolaise pour la

525 Conservation de la Nature (ICCN) for granting permission to conduct research at Salonga

526 National Park (MIN.0242/ICCN/DG/GMA/013/2013). The methods used to collect

527 observational data in the field are in compliance with the requirements and guidelines of

528 the ICCN and adhere to the legal requirements of the host country, the Democratic Republic 
529 of Congo. We are very grateful to Isaac Schamberg for his support in the field, to Barbara

530 Fruth for her collaboration in supporting our research at Lui Kotale Bonobo Project and to

531 members of Lompole village and all local staff supporting Lui Kotale. We are grateful to all

532 members of the Department of Comparative Cognition at the University of Neuchatel for

533 stimulating discussion, and especially to Christof Neumann for on-going support and

534 advice. We thank Jennifer Vonk, Adriano Lameria, Heidi Lynn and an anonymous reviewer

535 for their highly insightful comments on the manuscript.

536

537 References

538

Ackermann H, Hage SR, \& Ziegler W. (2014). Brain mechanisms of acoustic communication in humans and nonhuman primates: An evolutionary perspective. Behavioral and Brain Sciences, 37(06): 529-546.

Austin JL. (1975). How to do things with words (Vol. 367). Oxford: Oxford University Press.

Bates E, Benigni L, Bretherton I, Camaioni L \& Voltera V. (1979). The emergence of symbols: Cognition and communication in infancy. New York: Academic Press.

Bermejo M, \& Omedes A. (1999). Preliminary vocal repertoire and vocal communication of wild bonobos (Pan paniscus) at Lilungu (Democratic Republic of Congo). Folia Primatologica, 70(6): 328-357.

Boissy A, Manteuffel G, Jensen MB, Oppermann MR, Spruijit B, Keeling LJ, Winckler C, Behaviour 92(3): 375-397. 
552 Briefer EF \& McElligott AG. (2013). Rescued goats at a sanctuary display positive mood 553 after former neglect. Applied Animal Behavioral Science 146(1):45-55

554 Briefer EF, Tettamanti F \& McElligott AG. (2015). Emotions in goats: mapping physiological, 555 behavioural and vocal profiles. Animal Behaviour, 99: 131-143.

Bryant GA, \& Aktipis CA. (2014). The animal nature of spontaneous human laughter. Evolution and Human Behavior, 35(4): 327-335.

Clay Z, Ravaux L, de Waal FBM, Zuberbuhler, K. Under Review. Bonobos vocally protest to violations of social expectations.

Clay Z, \& Zuberbühler K. (2009). Food-associated calling sequences in bonobos. Animal Behaviour, 77(6): 1387-1396.

562

Clay Z, \& Zuberbühler K. (2011). Bonobos extract meaning from call sequences. PLoS One, 563 6(4): e18786.

564

Crockford C, Herbinger I, Vigilant L, \& Boesch C. (2004) Wild chimpanzees produce group565 specific calls: a case for vocal learning? Ethology 110(3): 221-243.

Crockford C, Wittig RM, Mundry R, \& Zuberbühler K. (2012). Wild chimpanzees inform ignorant group members of danger. Current Biology, 22(2): 142-146.

Da Costa AP, Leigh AE, Man M-S, \& Kendrick KM. (2004) Face pictures reduce behavioural, autonomic, endocrine and neural indices of stress and fear in sheep. Proceedings of the Royal Society of London B: Biological Sciences 271(1552): 2077-2084.

Davies A, Radford A, \& Nicol C. (2014) Behavioural and physiological expression of arousal during decision-making in laying hens. Physiology and Behaviour 123:93-99. compared to that of chimpanzees. Behaviour, 106(3-4): 183-251. 
575 Ekman P. (1992) Are there basic emotions? Psycholgical Review, 99: 550-553

576 Fichtel C, \& Hammerschmidt K. (2001). On the vocal expression of emotion. A multiparametric analysis of different states of aversion in the squirrel monkey. Behaviour 138 (1): 97-116.

Fitch WT. (2006). Production of vocalizations in mammals. Visual Communication, 3: 145.

580

581

582

583

584

585

586

587

588

589

590

591

592

593

594

595

596

Fitch WT, \& Hauser MD. (1995). Vocal Production in Nonhuman-Primates - Acoustics, physiology, and functional constraints on honest advertisement." American Journal of Primatology, 37(3): 191-219.

Greenfield PM, \& Savage-Rumbaugh ES. (1990). Grammatical combination in Pan paniscus: Processes of learning and invention in the evolution and development of language. In S. T. Parker \& K. R. Gibson, eds. 'Language' and intelligence in monkeys and apes. Cambridge: Cambridge University Press, 540-578.

Greenfield PM, \& Savage-Rumbaugh, ES. (1993). Comparing communicative competence in child and chimp: the pragmatics of repetition. Journal of Child Language, 20(01): 126.

Griebel U, \& Oller D. (2008) Evolutionary forces favoring communicative flexibility. In: Oller DK, Griebel U, eds. Evolution of communicative flexibility: Complexity, creativity, and adaptability in human and animal communication. Cambridge, MA: MIT Press, 9-40.

Gruber T, \& Zuberbühler K. (2013) Vocal Recruitment for Joint Travel in Wild Chimpanzees. PloS One 8(9):e76073.

Hage SR, Gavrilov N, \& Nieder A. (2013). Cognitive control of distinct vocalizations in rhesus monkeys. Journal of Cognitive Neuroscience, 25(10): 1692-1701. 
597 Hardus ME, Lameira AR, Van Schaik CP, \& Wich SA. (2009). Tool use in wild orang-utans 598 modifies sound production: a functionally deceptive innovation? Proceedings of the $599 \quad$ Royal Society B: Biological Sciences, 1027.

600 Hopkins WD, \& Savage-Rumbaugh ES. (1991). Vocal communication as a function of 601 differential rearing experiences in Pan paniscus: A preliminary report. International 602 Journal of Primatology, 12(6): 559-583.

603 Hopkins WD, Taglialatela JP, \& Leavens DA. (2007). Chimpanzees differentially produce 604 605 novel vocalizations to capture the attention of a human. Animal Behaviour, 73(2): 281-286.

Hotchkin CF, Parks SE, \& Weiss DJ. (2013). Vocal modifications in primates: Effects of noise and behavioral context on vocalization structure. Proceedings of Meetings on Acoustics (Acoustical Society of America), p 010061.

Imfeld-Mueller S, Van Wezemael L, Stauffacher M, Gygax L, \& Hillmann E. (2011) Do pigs distinguish between situations of different emotional valences during anticipation? Applied Animal Behavioural Science 131(3):86-93.

612 Janik V. (2000). Food-related bray calls in wild bottlenose dolphins (Tursiops truncatus). 613 Proceedings of the Royal Society of London. Series B: Biological Sciences, 267(1446): 923-927.

615 Koda H, Lemasson A, Oyakawa C, Pamungkas J, \& Masataka N. (2013). Possible role of 616 mother-daughter vocal interactions on the development of species-specific song in gibbons. PloS One, 8(8): e71432. 
618 Lameira AR, Hardus ME, Bartlett AM, Shumaker RW, Wich SA, \& Menken SB. (2015).

619 Speech-Like Rhythm in a Voiced and Voiceless Orangutan Call. PloS One, 10(1):

$620 \quad$ e116136.

621 Lameira AR, Hardus ME, Nouwen KJ, Topelberg E, Delgado RA, Spruijt BM, Wich SA. (2013).

622 Population-Specific Use of the Same Tool-Assisted Alarm Call between Two Wild

623 Orangutan Populations (Pongopygmaeus wurmbii) Indicates Functional

$624 \quad$ Arbitrariness. PloS One, 8(7): e69749.

625 Laporte MN, \& Zuberbühler K. (2010) Vocal greeting behaviour in wild chimpanzee 626 females. Animal Behaviour 80(3):467-473.

627 Lemasson A, Hausberger M, \& Zuberbühler K. (2005). Socially meaningful vocal plasticity in 628 adult Campbell's monkeys (Cercopithecus campbelli). Journal of Comparative 629 Psychology, 119(2): 220.

630 Lemasson A, Ouattara K, Petit EJ, \& Zuberbühler K. (2011). Social learning of vocal 631 structure in a nonhuman primate? BMC Evolutionary Biology, 11(1): 362.

632 Liebal K, Waller BM, Slocombe KE, \& Burrows AM. (2013) Primate Communication: a 633 multimodal approach. Cambridge: Cambridge University Press.

634 Locke JL, \& Bogin B. (2006). Language and life history: A new perspective on the 635 development and evolution of human language. Behavioral and Brain Sciences,

636 29(3): 259-279.

637 Macedonia JM, \& Evans CS. (1993). Variation among mammalian alarm call systems and the 638 problem of meaning in animal signals. Ethology, 93: 177-197.

639 Mateo JM. (2010) Alarm calls elicit predator-specific physiological responses. Biology 640 Letters :rsbl20100118. 
641 Mendl M, Burman OH, Parker RM, \& Paul ES. (2009) Cognitive bias as an indicator of animal 642 emotion and welfare: emerging evidence and underlying mechanisms. Applied 643 Animal Behavioral Science 118(3):161-181.

644 Mendl M, Burman OH, \& Paul ES. (2010) An integrative and functional framework for the 645 study of animal emotion and mood. Proceedings of the Royal Society B: Biological 646 Sciences 277(1696):2895-2904.

647 Mundry R, \& Sommer C. (2007). Discriminant function analysis with nonindependent data: 648 consequences and an alternative. Animal Behaviour, 74(4): 965-976.

649 Nettle D, \& Bateson M. (2012) The evolutionary origins of mood and its disorders. Current 650 Biology 22(17):R712-R721.

651 Newman JD. (1985). The infant cry of primates. In: Lester BM, Zachariah Boukydis CF., eds., 652 Infant crying. Springer:New York, 307-323.

653 Notman H, \& Rendall D. (2005). Contextual variation in chimpanzee pant hoots and its 654 implications for referential communication. Animal Behaviour, 70, 177-190.

655 Oller DK. (2000). The emergence of the speech capacity. Mahwah, NJ: Lawrence Erlbaum $656 \quad$ Associates.

657 Oller DK, Buder EH, Ramsdell HL, Warlaumont AS, Chorna L, \& Bakeman R. (2013).

658 Functional flexibility of infant vocalization and the emergence of language. 659 Proceedings of the National Academy of Sciences, 110(16), 6318-6323.

660 Ouattara K, Lemasson A, \& Zuberbühler K. (2009). Campbell's monkeys concatenate 661 vocalizations into context-specific call sequences. Proceedings of the National $662 \quad$ Academy of Sciences, 106(51), 22026. 
663 Owren MJ, \& Amoss RT. (2014). Spontaneous human laughter. In: Tugade MM, Shiota, MN, 664 Kirby LD, eds. Handbook of Positive Emotions. New York: Guildford Press, 159-178.

665 Owren MJ, Amoss RT, \& Rendall D. (2011). Two organizing principles of vocal production: 666 Implications for nonhuman and human primates. American Journal of Primatology, 667 73(6), 530-544.

668

Owren MJ, Seyfarth RM \& Cheney DL. (1997). The acoustic features of vowel-like grunt 669 calls in chacma baboons (Papio cynocephalus ursinus): implications for production processes and functions. Journal of the Acoustical Society of America, 101: 29512963.

Panksepp J. (1998) Affective neuroscience: The foundations of human and animal emotions. New York, NY: Oxford University Press.

674 Parr LA, Waller BM, \& Heintz M. (2008). Facial expression categorization by chimpanzees 675 using standardized stimuli. Emotion, 8(2): 216.

Parr LA, Waller BM, \& Vick SJ. (2007). New developments in understanding emotional facial signals in chimpanzees. Current Directions in Psychological Science, 16(3), 117-122.

Perlman M, Patterson FG, \& Cohn RH. (2012). The human-fostered gorilla Koko shows breath control in play with wind instruments. Biolinguistics, 6(3-4): 433-444.

Reefmann N, Wechsler B, \& Gygax L. (2009) Behavioural and physiological assessment of 681 positive and negative emotion in sheep. Animal Behaviour 78(3): 651-659.

Rendall D. (2003) Acoustic correlates of caller identity and affect intensity in the vowel-like 683 grunt vocalizations of baboons. Journal of the Acoustical Society of America, 113 (6):

684 3390-3402 
685 Rendall, D, Seyfarth, RM, Cheney DL \& Owren, MJ. (1999) The meaning and function of 686 grunt variants in baboons. Animal Behaviour 57: 583-592.

687 Russell JA. (1980). A circumplex model of affect. Journal of Personality and Social 688 Psychology, 39(6):1161

689 Russell JA \& Barrett LF. (1999) Core affect, prototypical emotional episodes, and other 690 things called emotion: dissecting the elephant. Journal of Personality and Social Psychology, 76(5):805.

692

693

694

695

696

697

698

699

700

701

702

703

704

705

706

Savage-Rumbaugh ES. (1988). A new look at ape language: Comprehension of vocal speech and syntax. Comparative perspectives in modern psychology. In D. W. Leger, ed. Nebraska Symposium on Motivation. Lincoln, NB: University of Nebraska Press. Vol. $35,201-256$.

Savage-Rumbaugh ES. (1990). Language acquisition in a nonhuman species: Implications for the innateness debate. Special Issue: The idea of innateness: Effects on language and communication research. Developmental Psychobiology, 23(7), 599-620.

Savage-Rumbaugh ES, Shanker SG, \& Taylor TJ. (1998). Apes, language, and the human mind. New York: Oxford University Press.

Savage-Rumbaugh ES, \& Lewin R. (1994). Kanzi. The ape at the brink of the human mind. New York: Wiley.

Scarantino A, \& Clay Z. (2014). Contextually variable signals can be functionally referential. Animal Behaviour, 100: 1-8.

Schel AM, Machanda Z, Townsend SW, Zuberbühler K, \& Slocombe KE. (2013) Chimpanzee food calls are directed at specific individuals. Animal Behaviour 86(5): 955-965. 
707 Schel AM, Townsend SW, Machanda Z, Zuberbühler K, \& Slocombe KE. (2013) Chimpanzee

708 alarm call production meets key criteria for intentionality. PloS ONE 8(10):e76674.

709 Segerdahl P, Fields W, \& Savage-Rumbaugh ES. (2005). Kanzi's primal language: The

$710 \quad$ cultural initiation of primates into language.New York:Palgrave Macmillan.

711 Seyfarth RM, Cheney DL, \& Marler P. (1980). Vervet monkey alarm calls: Semantic

712 communication in a free-ranging primate. Animal Behaviour 28: 1070-1094.

713 Seyfarth RM, Cheney DL, \& Marler P. (1980). Monkey responses to three different alarm

714 calls: evidence for predator classification and semantic communication." Science,

210: 801-803.

716 Slocombe KE, Kaller T, Turman L, Townsend SW, Papworth S, Squibbs P,

717

718

719

720

721

722

723

724

725

726

727

728

Zuberbühler K. (2010). Production of food-associated calls in wild male chimpanzees is dependent on the composition of the audience. Behavioural Ecology and Sociobiology 64(12):1959-1966.

Slocombe KE, \& Zuberbühler K. (2007). Chimpanzees modify recruitment screams as a function of audience composition. Proceedings for the National Academy of Sciences of the United States of America, 104, 17228-17233.

Tabachnick BG \& Fidell LS. (2001) Using multivariate statistics. Boston, MA: Pearson.

Taglialatela JP, Reamer L, Schapiro SJ, \& Hopkins WD. (2012). Social learning of a communicative signal in captive chimpanzees. Biology Letters, 8(4), 498-501.

Taglialatela JP, \& Savage-Rumbaugh S. (2003). Vocal production by a language-competent Pan paniscus. International Journal of Primatology, 24(1), 1-17.

Tomasello M. (2008). Origins of human communication. Cambridge, MA: MIT Press. 
729 Tomasello M, \& Carpenter M. (2007). Shared intentionality. Developmental Science, 10(1):

730 121-125.

731 Tomasello M, Carpenter M, Call J, Behne T, \& Moll H. (2005). Understanding and sharing 732 intentions: The origins of cultural cognition. Behavioral \& Brain Sciences, 28, 1-17.

733

734

735

736

737

738

739

740

741

742

743

744

745

746

747

748

749

750

751

Townsend SW, Deschner T, \& Zuberbuehler K. (2008) Female chimpanzees use copulation calls flexibly to prevent social competition. Plos ONE 3(6).

Van Krunkelsven E, Dupain J, Van Elsacker L, \& Verheyen RF. (1996). Food calling in captive bonobos (Pan paniscus): an experiment. International Journal of Primatology, 17, 207-217.

Watson D, Wiese D, Vaidya J, \& Tellegen A. (1999) The two general activation systems of affect: Structural findings, evolutionary considerations, and psychobiological evidence. Journal of Personality and Social Psychology, 76(5):820.

Wich SA, Swartz KB, Hardus ME, Lameira AR, Stromberg E, \& Shumaker RW. (2009). A case of spontaneous acquisition of a human sound by an orangutan. Primates, 50(1), 5664.

Wilkinson GS, \& Boughman WJ. (1998). Social calls coordinate foraging in greater spearnosed bats. Animal Behaviour, 55(2): 337-350.

Zuberbühler K. (2003). Referential signaling in non-human primates: Cognitive precursors and limitations for the evolution of language. Advances in the Study of Behavior: 33, 265-307.

Zuberbühler K. (2006). Alarm calls - Evolutionary and cognitive mechanisms. In K. Brown (Ed.), Encyclopedia of Language and Linguistics (2 ed., pp. 143-155). Oxford: 
Figure 1 (on next page)

Figure 1

Figure 1. Time-frequency spectrograms illustrating peeps produced by four wild bonobos (BE, $\mathrm{EM}, \mathrm{ZD}=$ male, $\mathrm{NI}=$ female) during different behavioural contexts. The emotional valence of the context is indicated in parantheses. 


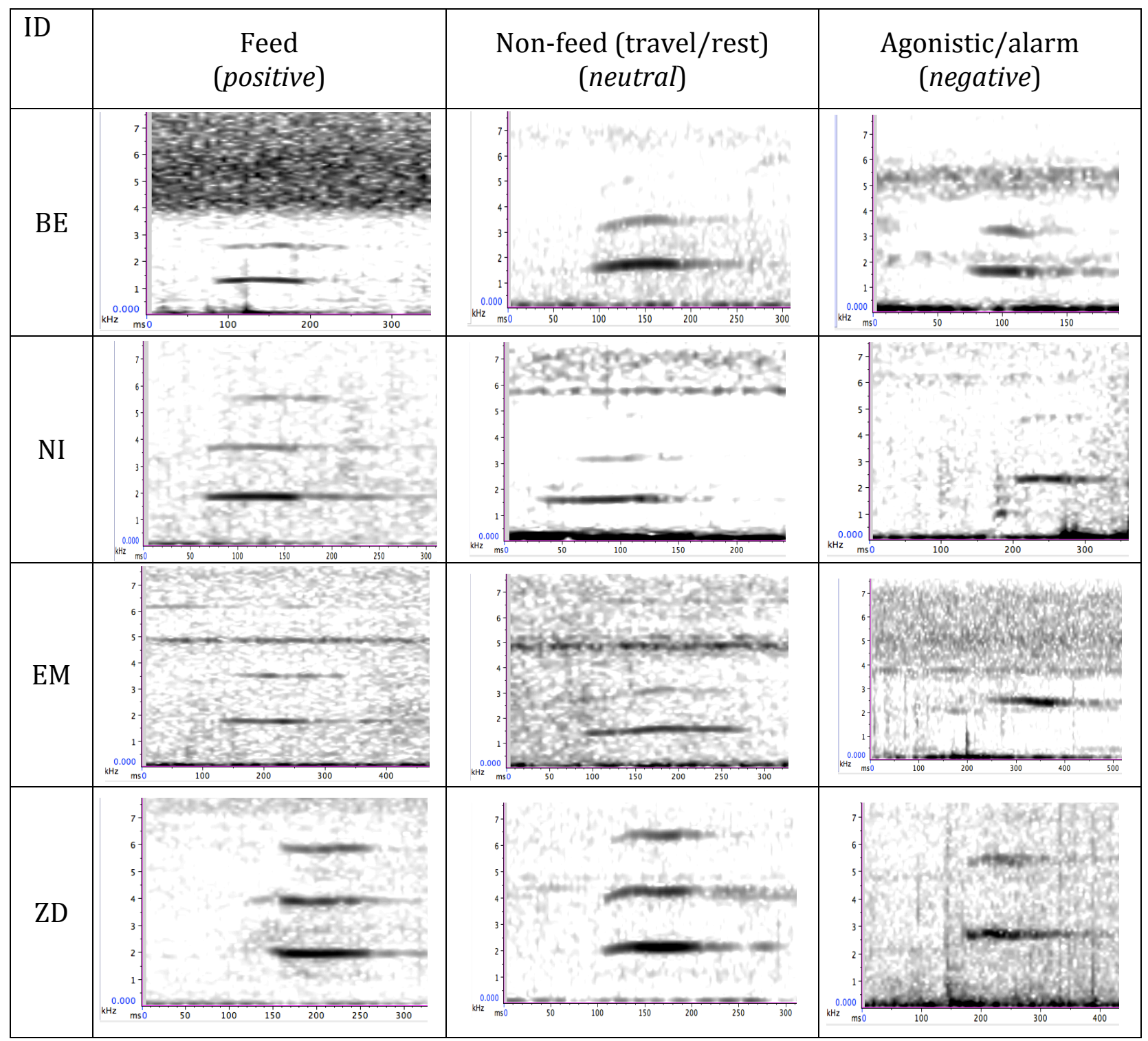


Figure 2 (on next page)

Figure 2

Figure 2. Boxplots indicating six acoustic parameters of peep vocalisations that varied as a function of behavioural context. The emotional valence associated with the context is indicated in parentheses. Thick black lines represent medians; open circles and small asterisks represent outliers, box edges represent the upper and lower hinges of the $\mathrm{H}$ spread, which generally matches the upper and lower quartiles; whiskers represent the adjacent values, which are the most extreme values still lying within hinges and the normal distribution of the sample. For significant differences, lines with ** represents $\mathrm{P}<.05$, *** represents $\mathrm{P}<.001$. 

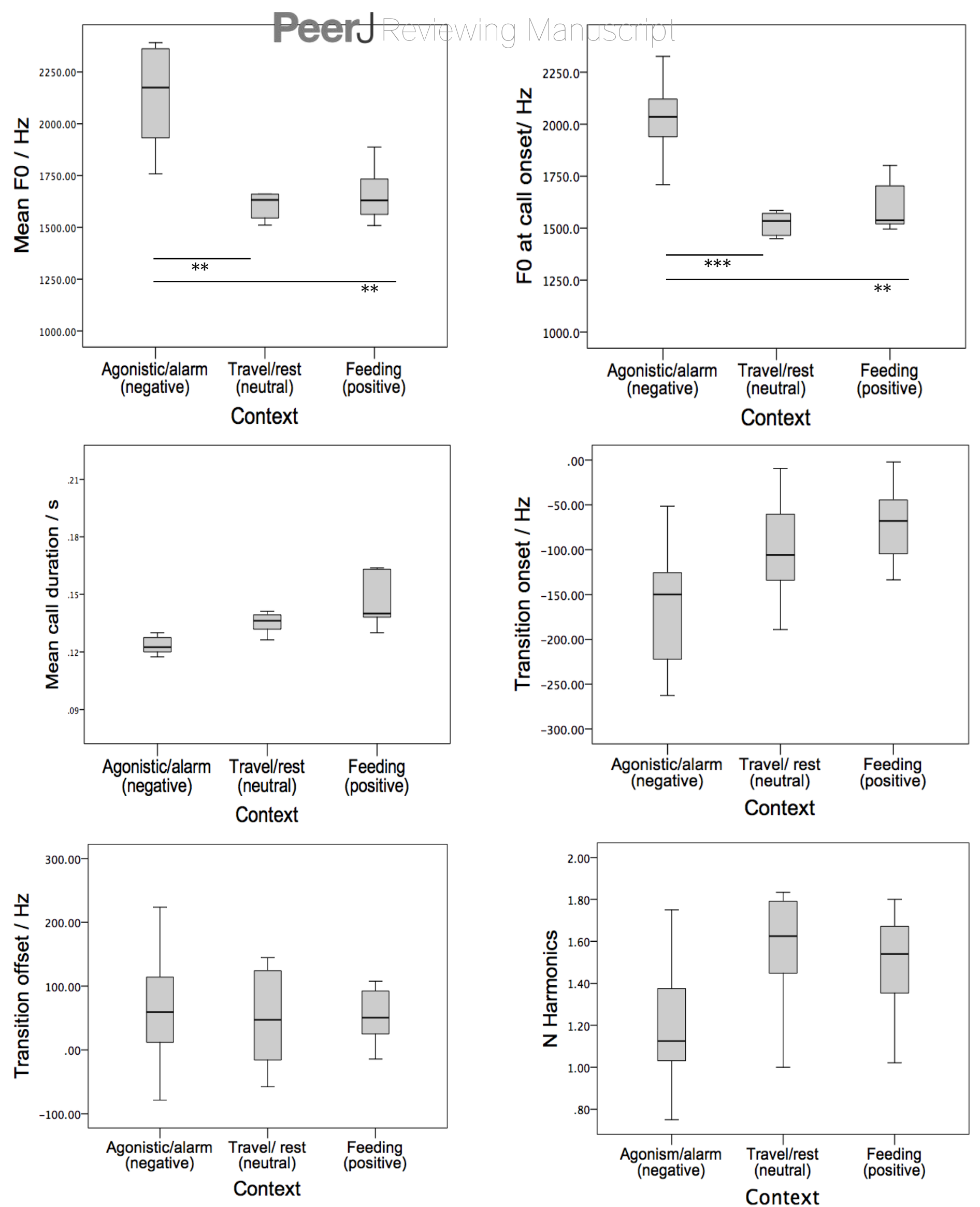